\title{
TURISMO DE CRUCEROS EN LA INTERFAZ PUERTO-CIUDAD MEDITERRÁNEA: NUEVA ÉPOCA DEL WATERFRONT, CON BENEFICIOS EN UN SMART DESTINATION
}

\author{
Beatriz Perea-Medina* \\ María J. Andrade** \\ Carlos Rosa-Jiménez** \\ Universidad de Málaga
}

\section{RESUMEN}

Este estudio se sitúa en la literatura sobre la industria de cruceros atendiendo al fenómeno waterfront y al desarrollo de una nueva interfaz crucero-ciudad promovida por el turismo de cruceros. Se parte de una hipótesis, el desarrollo de una nueva época del waterfront con la implicación del turismo de cruceros, que incide tanto en el waterfront como en el puerto, bajo el desarrollo de una nueva imagen como puerta de entrada a la ciudad, y la ciudad mediterránea, principalmente su casco histórico, al ser el área cultural atrayente, que forman parte de las intervenciones en la búsqueda de la relación puerto-ciudad. En este sentido, se ponen de manifiesto los impactos positivos y negativos que trae consigo este tipo de turismo, tras estudiar diferentes casos de ciudades portuarias mediterráneas y se concluye, con la propuesta de una integración a través del desarrollo de un Smart Cruise Port en un Smart Destination, que beneficiaría la interrelación crucero-ciudad.

Palabras clave: turismo de cruceros; transformaciones en waterfront; interfaz puertociudad mediterránea; Destino Turístico Inteligente; Puerto de Cruceros Inteligente.

Fecha de recepción: 25 de marzo de 2017.

Fecha de aceptación: 17 de octubre de 2017.

* Universidad de Málaga, Andalucía Tech, Escuela Politécnica Superior. Campus de Teatinos s/n. 29071 MÁLAGA (España).E-mail: bpmarquitecto@hotmail.com

** Habitat-Tourism-Territory Institute, Universidad de Málaga. Universidad Politécnica de Cataluña. Edificio Ada Byron. Campus de Teatinos, s/n. 29071 MÁLAGA (España).E-mail: mjandrade@uma.es, cjrosa@uma.es 


\title{
Cruise tourism in the Mediterranean port-city interface: new era of the waterfront, with benefits in a Smart Destination.
}

\begin{abstract}
This study is part of the literature on the cruise industry that focuses on the waterfront phenomenon and on the development of a new cruise-city interface promoted by cruise tourism. The development of a new era of waterfronts with the implication of cruise tourism is used as the starting hypothesis. It affects both the waterfront and the port, under the development of a new image as a gateway to the city and Mediterranean cities (mainly their historical downtown, since it is the attractive cultural area) which are part of the interventions in the search for the port-city relationship. In this sense, the positive and negative impacts of this type of tourism are listed after studying different cases of Mediterranean port cities. The integration through the development of a Smart Cruise Port in a Smart Destination, which would benefit the cruise-city interrelationship, is presented as a proposal.
\end{abstract}

Keywords: cruise tourism; waterfront transformations; Mediterranean cruise-city interface; Smart Tourist Destination; Smart Cruise Port.

\section{INTRODUCCIÓN}

"Después de más de treinta experiencias exitosas en todo el mundo, cada vez más ciudades y puertos se plantean proyectos de revitalización de sus fachadas marítimas, podríamos decir que un proyecto de este tipo es integral, dado que por un lado, la revitalización de los viejos espacios portuarios cercanos al centro de la ciudad es una gran oportunidad de negocio para los agentes marítimos, los profesionales, las empresas turísticas, en definitiva, para el conjunto de la ciudad, y por otro lado, nuevos tráficos portuarios que aportan actividad y riqueza al puerto y a la ciudad (marinas, pasajeros, cruceros...) necesitan entornos portuarios de mayor calidad urbanística y de buenas relaciones con la ciudad." (Alemany, 1984).

La industria de cruceros es uno de los subsectores turísticos más dinámicos y de mayor expansión de los segmentos de la industria del turismo internacional (Sun et al., 2011), por lo que si los destinos quieren mantener o formar parte de esta industria, deben transformarse y adaptarse a los condicionantes que trae consigo, dado que el desarrollo de buques cada vez más grandes, provoca que muchos destinos turísticos se enfrenten no sólo a problemas de cabida a estos mega buques, sino a problemas sociales, ambientales e infraestructurales que afectan al destino. No obstante, también conlleva impactos positivos como mejoras urbanísticas, beneficios económicos que llegan a extenderse a nivel regional o creación de empleo.

El fin del crucero es intentar mantener a bordo al mayor número de clientes durante el mayor tiempo posible con el fin de generar más beneficios -reducción del coste de las bebidas, de los souvenirs en las tiendas de abordo y de actividades de pago como el acceso al spa, durante la parada en un puerto de escala- frente a las estrategias de marketing llevadas a cabo por la ciudad para atraer al pasajero de cruceros. 
En cualquier caso, el turismo de cruceros que se ofrece en las ciudades portuarias mediterráneas, necesita la ciudad no sólo para el repostaje o el repuesto de suministros, sino también porque la oferta como un atractivo más del viaje en crucero. Por tanto, este tipo de turismo, en el Mediterráneo, tras la reconversión de sus ciudades portuarias, tiene implicaciones directas en el puerto, el waterfront y la ciudad (Figura 1) como se verá a continuación.

\section{Figura 1 \\ EJEMPLO DE TRANSFORMACIÓN. PUERTO DE MÁLAGA}

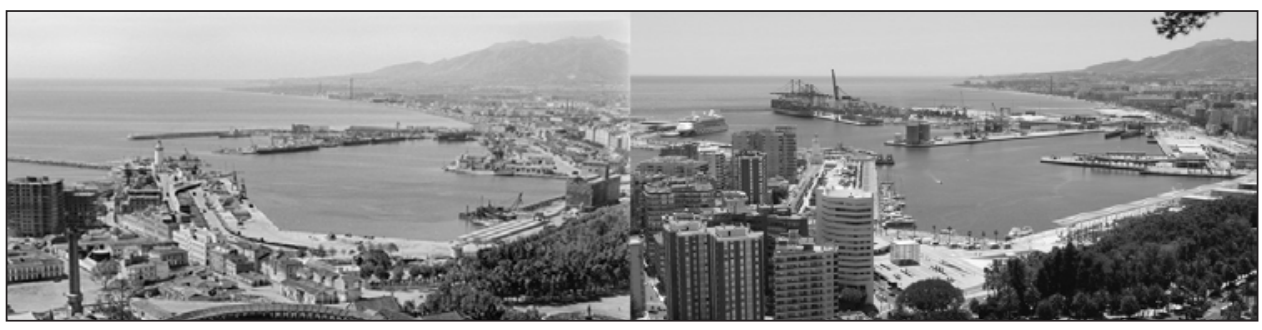

Fuente: http://archivocti.uma.es/icaatom/index.php/malaga-vista-de-la-malagueta-plaza-de-toros-y-puerto-desdela-alcazaba-abril-de-1963;dc (imagen izquierda); http://www.malagaport.eu/6183/ (imagen derecha).

En primer lugar se encuentra el puerto, que para permitir el acceso de cruceros requiere ciertas instalaciones. Las principales infraestructuras necesarias son muelles y estaciones marítimas. Los muelles, permiten el atraque de los buques, unos se extienden hacia el mar (p. e. Puerto de Málaga), otros a lo largo de su costa (p. e. Puerto de Marsella) y en ciertos casos, aunque haya puerto, si el destino potencial se encuentra alejado de éste, el barco fondea (p. e. Santorini). Las estaciones marítimas, por su parte, no sólo se instalan para gestionar el tránsito de los pasajeros, sino que están íntimamente relacionadas con la creación de una nueva imagen de la ciudad al implantar edificios "de autor" como nuevas puertas de la ciudad.

En cuanto al waterfront, entendido como un área estratégica de las ciudades portuarias (Pavia, 2011) que impulsan a la modernización de los cascos históricos como nuevo foco de centralidad donde discurren vida urbana y marítima, el transporte de pasajeros produjo un nuevo acceso a la ciudad por el mar, por lo que los frentes marítimos de las ciudades portuarias se han visto envueltos en procesos de reutilización, renovación y regeneración, conformando un nuevo skyline, visualizado desde la entrada al puerto. Además, es un factor clave su aportación urbanística, tanto en el desarrollo urbanístico y económico, donde se tienen en cuenta las importantes obras de arquitectura que han facilitado mejorar dichas áreas y hacerlas partícipes de la ciudad, como es el caso de la Ópera de Sydney (Jorn Utzon) en el escenario marítimo o el Guggenheim (Frank Gehry) en el fluvial, dado que la imagen juega un factor relevante, tanto en el propio ciudadano, como en el turista, bien para la elección de destino o como influencia en el comportamiento de viajes posteriores (Barroso et al., 2007), así como en la ordenación del territorio, que debe ser tratado, no como el espacio existente entre el puerto y la ciudad, sino como área confluyente e indivisible de las dos zonas donde la conexión puerto-ciudad sea fluida, permeable e imposible de delimitar. 
Por último, la ciudad portuaria mediterránea, la cual es uno de los motivos principales del pasajero de cruceros, pues en muchos casos, eligen un crucero por los destinos que ofrece en su itinerario. En este caso, dichas ciudades son atrayentes por sus elementos culturales, que generalmente se ubican en los centros históricos (por ejemplo, Málaga), es el propio centro histórico, en su totalidad, el recurso cultural (p. e. Dubrovnik) e incluso la propia ciudad (p. e. Venecia). En este sentido, se hace relevante que el casco antiguo se encuentre próximo al puerto, dado que los cruceristas tienen un tiempo limitado para visitar la ciudad, pero no es una característica importante extrapolable a todos los destinos, porque en el caso de los puertos dependientes, como Civitavecchia de Roma, los cruceristas recorren 80 kilómetros para llegar a la ciudad objeto de visitar.

En este artículo, se aborda el turismo de cruceros como elemento importante en la reactivación de las tres partes - puerto, waterfront y ciudad- pero sin tratarlos independientemente, de forma parcial, sino como un todo, dado que las infraestructuras ocasionadas en el waterfront, que dan cabida a las actividades terciaria y cultural, no sólo forman parte de ese puerto en constante transformación para permitir el atraque de los buques de crucero, sino que en muchos casos, como el Moll de la Fusta, en Barcelona, es a su vez, la propia fachada de una ciudad que se transforma para dar cabida a los pasajeros de cruceros, principalmente, en su casco histórico.

Por tanto, se tratará, por un lado, el waterfront como foco de interrelación entre las diferentes partes, cuyas transformaciones, ocasionadas en un primer momento por los cambios industriales, se han convertido en un punto focal en la planificación y el desarrollo urbanístico de las ciudades marítimo-portuarias y por otro lado, el casco histórico, como área que se reactiva en la búsqueda de acercamiento al mar, a través de la incorporación de equipamientos comerciales y culturales, ofreciendo así, una expansión del propio waterfront, trayendo consigo una mejora de su conexión.

Existen pocos estudios sobre las transformaciones ocasionadas en el waterfront. Norcliffe, Bassett y Hoare (1996), estudian los procesos de evolución y desarrollo del puerto y la ciudad, llegando al periodo de aparición del waterfront como área de oportunidad para la creación de empleo, viviendas, ocio y cultura. Ese mix de usos, generan un "place experiences" y un nuevo nicho de consumo en la economía local.

En general, los estudios se centran (a) en casos individuales de regeneración del frente marítimo, (b) en propuestas para potenciarlos o (c) en una búsqueda de aceptación por parte de los ciudadanos o de las autoridades gubernamentales, planificadores y promotores.

(a) Los primeros estudios se realizan para las remodelaciones de costa desarrollados en Estados Unidos: Toronto (Laidley, 2007); Portland, Oregón (Hagerman, 2007); Kingston, Jamaica (Dodman, 2007); Hamilton, Canadá (Wakefield, 2007); Portsmouth, Reino Unido (Cook, 2004), posteriormente, aparecen estudios que incorporan las transformaciones europeas en estos espacios, como el de Harms (2008), que compara el desarrollo de un waterfront estadounidente, el de San Francisco, con uno europeo, el de Hamburgo y, finalmente, ciertos estudios tratan los waterfront de ciudades portuarias europeas y mediterráneas como el de Gunay y Dokmeci (2012), estudiando la regeneración del frente marítimo de Cuerno de Oro (Estambul) como proyecto que puede conducir al crecimiento y expansión de la cultura 
turca, así como a una mejora de la imagen de la ciudad o como el de Daamen y Vries (2013), que definen los cambios espaciales producidos en la línea de costa de las ciudades portuarias junto con los procesos institucionales que dan forma a esos cambios, estudiando los proyectos ejecutados en la interfaz puerto-ciudad en los casos de Marsella, Barcelona, Hamburgo y Rotterdam.

(b) En el segundo estudio se sitúa Gospodini (2001) planteando posibles rediseños de los frentes marítimos de las ciudades portuarias griegas para mejorar su imagen, aumentar la calidad del entorno construido potenciando la relación entre el mar y la ciudad y desarrollar el turismo urbano.

(c) Por último, entre los estudios sobre el desarrollo del waterfront que muestran las preferencias y actitudes de usuarios o expertos, se sitúa Hoyle (1999), tratando los cambios producidos en el frente marítimo de las ciudades portuarias canadienses de Halifax, Kingston, St John's, Vancouver y Victoria, basándose en las experiencias, críticas e ideas de grupos o comunidades locales y posteriormente, dicho autor en 1994, trata las actitudes y percepciones del desarrollo de los waterfronts en las ciudades portuarias canadienses a través de los representantes de las autoridades portuarias, los urbanistas y los promotores. En cuanto a las ciudades portuarias mediterráneas, Vayona (2011), estudia las posibles remodelaciones del frente marítimo de Thessaloniki (Grecia) a través de las preferencias de sus ciudadanos.

Por tanto, dentro de la literatura existente en el campo de la industria del turismo de cruceros, este documento se presenta como una continuación a los estudios del waterfront donde interviene el turismo de cruceros como eje canalizador, en ciertas ocasiones, de las relaciones puerto-ciudad. Siendo la hipótesis de partida, el estudio de una nueva época del waterfront, en el cual se integran actividades de cruceros, para generar o mejorar la unión entre el puerto y la ciudad, combinando y maximizando las actividades del frente marítimo con los pasajeros de cruceros, los residentes y los visitantes, así como con el casco histórico de la propia ciudad para desarrollar una red interconectada puerto-ciudad y viceversa.

\section{METODOLOGÍA}

Para demostrar la hipótesis, en primer lugar, se expondrán las cuatro etapas del waterfront (Andrade Marqués, 2012; Brownill, 2013), como espacio generado tras los avances puerto-ciudad y situado en esa última fase de acercamiento e integración urbana-portuaria (Hoyle, 1998), ocasionado por la globalización y el nuevo papel de los puertos, cuyos espacios infrautilizados son convertidos en oportunidad.

En segundo lugar, se propondrá y evaluará una quinta etapa como evolución de la anterior donde se incluye el turismo de cruceros, dentro de la interfaz crucero-puertowaterfront-ciudad, en adelante interfaz crucero-ciudad, dado que favorece y formaliza las demandas de nuevas infraestructuras marítimo-terrestre y se estudiará el casco histórico como continuación del waterfront enfocado al turismo de cruceros, fomentando y promoviendo una conexión real con el puerto no sólo a través de equipamientos, sino incluyendo diferentes factores (Kwortnik, 2008) como los ambientales -olores, sonidos, 
imágenes- (por ejemplo, en Mykonos o Santorini, las imágenes generadas en calles del centro histórico desde donde se puede ver el mar y casi, se puede oler y sentir su brisa); los de diseño -arquitectura, decoración- (recuperación de sitios históricos, reconversión del patrimonio industrial abandonado, edificios de autor) y los factores sociales -participación activa de los residentes y de los actores sociales locales-.

A continuación, se realiza un estudio de casos centrado en ciudades portuarias mediterráneas, entendiendo el turismo de cruceros como factor que no ha formado parte de la transformación de la interfaz waterfront-ciudad (únicamente se expresa la interfaz de estos elementos dado que es evidente que las modificaciones del puerto las han llevado a cabo), como son Barcelona o Venecia; como un tipo más de turismo que ha influido en la reforma de la interfaz crucero-ciudad, como pueden ser destinos turísticos con un fuerte potencial cultural como Dubrovnik, donde los pasajeros de cruceros forman parte de ese número de turistas que recorren la ciudad; y, esta industria como principal motivo de transformación, llevando al destino a un gran cambio en la conexión mar-tierra como pueden ser Málaga o Marsella.

Por último, se propone el desarrollo de un Smart Cruise Port en un Smart Destination como alternativa a las mejoras de relación puerto-ciudad que mejoraría notablemente la interfaz crucero-ciudad.

\section{UNA NUEVA ETAPA EN LOS WATERFRONT: TURISMO DE CRUCEROS COMO ESTÍMULO DEL WATERFRONT I CENTRO HISTÓRICO}

\subsection{Evolución del Waterfront: Las cuatro grandes etapas}

Desde la segunda mitad del siglo XIX, se pueden distinguir dos tipos de acercamiento de la ciudad al mar (Griffin y Hayllar, 2006; Jones, 2007; Xie y Gu, 2015; Rosa-Jiménez y Perea-Medina, 2016) que promovidos por un pasado, donde los puertos favorecían el intercambio de mercancías, personas y culturas, quedaron obsoletos, probablemente por infraestructuras, diseño y/o accesibilidad inapropiadas generadas por las nuevas técnicas de la era industrial que requerían mayor extensión de terreno, así como aumento de las instalaciones existentes, donde en la mayoría de los casos serían trasladadas las funciones más pesadas como el almacenamiento de contenedores a zonas alejadas del puerto y la ciudad dejando en desuso grandes áreas de suelo y llegando a esa fase de separación y aislamiento de la ciudad con la aparición de "barreras" que distanciaban al ciudadano de su contacto con el agua, se han llegado a convertir en nuevos focos de centralidad urbana con intervenciones urbano-portuarias favoreciendo la intrínseca relación puerto-ciudad que había sido perdida, desarrollando una nueva fase de especialización de los puertos dentro de la etapa de acercamiento e integración ciudad-puerto.

Así, por un lado, se encuentra la marítima contemporánea relacionada con la actividad turística y del ocio, que facilitan el acceso al puerto, potenciando tanto la zona como las actividades en la idea de continuación de la trama urbana, así como la industria de cruceros, ésta última, representada como símbolo de la "industrialización en masa" en actividades de ocio y que ofrece un punto de vista particular de entender la evolución del turismo mundial (Sinclair y Stabler, 1997) dado que las líneas marítimas regulares que- 
daron en un segundo plano con la llegada del avión, más concretamente con los vuelos chárter, y los buques fueron destinados al nuevo servicio de transporte de pasajeros con fin recreativo, por lo que, de esta manera, se permitió la introducción del crucero como nuevo modelo de negocio y se empezó a contar con barcos más grandes e itinerarios más operativos y con un puerto que se ha ido adaptando a los cambios en el transporte marítimo con la introducción, modificación y ampliación de infraestructuras -muelles, terminales, estaciones marítimas con usos complementarios como restauración, comercios, áreas de exposición, aparcamientos- que ha formado parte del importante desarrollo urbano desde la segunda mitad del siglo XX.

Por otro, la portuaria antigua y tradicional, relacionada con el transporte y la actividad industrial y pesquera; que comienza a sufrir signos de obsolescencia desde mediados del siglo XX, debido a la desindustrialización o el cambio en las estructuras económicas, que llevó al abandono y deterioro de miles de hectáreas de suelo industrial portuario. Sin embargo, estos sectores urbanos en desuso, se convirtieron en oportunidad al ser áreas urbanas céntricas y estratégicas próximas al mar, que permitían no sólo renovar y reactivar dicha zona, sino que la transformación llegaría a extenderse más allá de sus límites, produciendo mejoras en el conjunto de la ciudad, en la trama urbana, en la interconexión tierra-mar, en el acercamiento al agua por parte del ciudadano, en la recuperación de la identidad local.

\section{Años 60: Reactivación económica del market place}

En esta etapa se sitúan puertos como el de Baltimore (Habour Place), Nueva York (Battery Park City), Boston (Quincy Market), el Pier 39 en San Francisco (Fisherman's Wharf) o Los Angeles (Ports of Call). El primero de ellos, el puerto de Baltimore, fue uno de los primeros puertos en ser renovados, que partiendo de un proyecto con tres fases: Charles Centre, Inner Habour y Market Centre, no sólo se convirtió en un modelo para los posteriores proyectos de remodelación de frentes marítimos, sino que mejoró la calidad de vida de los residentes, aumentó el número de turistas, creó numerosos puestos de trabajo e impulsó notablemente la economía (Millspaugh, 2003). En este contexto, se hace relevante referenciar el caso de Boston como pionero en la conservación y rehabilitación de edificios que marcó tendencias en los EE.UU. en general, al conservar dos naves próximas al puerto -Quincy Market y Faneuil Hall-y transformar antiguos almacenes en viviendas.

Estas primeras regeneraciones de frentes marítimos estaban basadas en la incorporación de instalaciones terciarias, equipamientos turísticos y de ocio, desplazando las actividades portuarias para desarrollar estas nuevas funciones, revalorizar ese emplazamiento y transformarlo en centro neurálgico de la ciudad.

\section{Años 80: Oficinas y residencia}

Hacia los años 80', se encontraría la segunda etapa, produciéndose un traslado de las experiencias americanas a Europa con nuevas propuestas caracterizadas por la variedad de usos y actividades, donde se incluye el residencial, dotando a los waterfront de carácter funcional. De este periodo, destaca el London Docklands Development Corporation 
(LDDC), más conocido como los London Docklands, en Londres, un proyecto de unas 3.000 hectáreas que abarcaba programas de vivienda y la creación de una red de transporte, cuyo papel fundamental era la revitalización de la línea de costa (Brownill, 1993) y la construcción de un mega-proyecto vinculado a la inversión económica mundial (Brownill, 2013), el Canary Wharf (1980-1990), un centro financiero con espacios públicos que se convirtió en el motor de la regeneración urbana de la ciudad.

\section{Años 90: Los eventos como economía}

La tercera etapa se sitúa en la década de los 90', donde los grandes eventos internacionales provocan en los waterfront una regeneración urbanística, convirtiéndose en "laboratorios urbanos" o "epicentros culturales" (Gospodini, 2006) de nuevos proyectos, en la búsqueda de una nueva imagen de la ciudad. Este es el caso de la experiencia precursora de Barcelona con motivo de la celebración de los Juegos Olímpicos en el 92', en el cual, se creó un Plan Estratégico para potenciar Barcelona como destino turístico internacional, o en Génova, con la exposición internacional “Cristóbal Colón: el barco y el mar” en 1992, comenzó el proceso de recuperación del waterfront, que continuó con el desarrollo de dos eventos: la Cumbre del G8 en 2001 y la elección de la ciudad como Capital Europea de la Cultura en 2004, que también incluía la transformación urbana del centro histórico y el paseo marítimo del Puerto Viejo (Gastaldi y Camerin, 2014).

De esta manera se observa, cómo el turismo, bajo la realización de festivales, celebraciones y/o eventos, enmarcado en la globalización, y el papel del patrimonio cultural (Roberts, 2000), influyen notablemente en la remodelación urbana jugando un papel fundamental las estrategias de marketing y planificación generadas en el waterfront, convirtiéndose así en un dispositivo que promueve la identidad del lugar y revitaliza espacios obsoletos.

\section{Años 00: Recuperación de la identidad social}

La siguiente evolución del waterfront, correspondiente a la última de las cuatro etapas, se centra en mantener o recuperar al máximo la actividad marítima -pesca, áreas deportivas- como símbolo distintivo del waterfront para promover una identidad que diferencie la ciudad portuaria de sus competidoras, combinándolo con la generación de usos ciudadanos que mantengan el puerto en la ciudad. En este sentido ha actuado Génova, al conservar las actividades de los transbordadores y cruceros en el Puerto Viejo, se ha generado un factor estratégico y positivo que salvaguarda la imagen urbana y la identidad del barrio (Gastaldi y Camerin, 2014).

Todas estas transformaciones han encontrado en el waterfront un espacio de representación a través de arquitecturas que se han diferenciado en dos, por el tipo de valor que puedan aportar. Por un lado, y en el mejor de los casos, edificios de gran valor arquitectónico y artístico que son utilizados como iconos de la ciudad para mostrar esa nueva imagen más propulsiva y dinámica, por otro, megaproyectos elitistas dirigidos a la búsqueda del máximo beneficio económico como centros comerciales, torres de oficinas, hoteles, centro de convenciones o edificios residenciales de lujo. 


\subsection{Actualización del Waterfront: formalización y adaptación de infraestructuras crucero-tierra}

Esta nueva etapa se presenta como otra evolución más del waterfront donde se une, lo procedente de las etapas anteriores -multitud de usos, piezas arquitectónicas que dan valor y generan una nueva imagen de la ciudad, donde se potencia el carácter portuario, proporcionando valor a las actividades marítimas- con el turismo de cruceros, como elemento principal de esta nueva fase al contar con una particularidad espacial que impulsa la reestructuración del espacio urbano. En muchos casos, el turismo de cruceros es sinónimo de mejoras infraestructurales tanto en el puerto como en la ciudad que permite la reactivación de los cascos históricos y estimula la unidad territorial al ofrecer ese acercamiento puerto-ciudad y viceversa. Además, es también un atributo social, al favorecer la conexión puerto-ciudad, económico, dado que beneficia la economía marítimo-portuaria y su función se alza a la de puerta de entrada internacional, adquiriendo funciones relacionadas con la globalización (Vallega, 2001).

Así, en esta etapa, se hace relevante definir el concepto turismo de cruceros, subsector turístico en situación de crecimiento, que ha demostrado su resistencia a la crisis y cuyas perspectivas de futuro son favorables, y los elementos que intervienen en él hasta llegar a la ciudad -crucero, puerto y terminal-.

El turismo de cruceros es entendido como un tipo de turismo producido por la unión entre la industria del ocio y del transporte marítimo, una unión llevada a cabo a principios del siglo XX y consolidada en los años cincuenta con la desaparición del tráfico marítimo de pasaje, que se concibe como un tipo de embarcación que ofrece relax, exclusividad, entretenimiento, calidad y permite descubrir gran cantidad de destinos mientras se viaja, además de añadir valor en la industria local. Este tipo de turismo está evolucionando y ampliando sus fronteras para acercarse a la clase media, contribuyendo con diversos factores como son, la realización de buques de mayor tamaño, mayor capacidad y por tanto, mayor número de pasajeros, planes de marketing y publicidad atrayentes para captar y mantener clientes, diversificación de las rutas con el consiguiente acercamiento a los potenciales clientes y no se puede obviar, el aumento del nivel de vida y del turismo en muchos países como puede ser la mayoría de los pertenecientes al continente africano.

El buque es un resort, un producto turístico complejo y segmentado que actúa de foco de atracción como principal destino, permitiendo de esta manera competir con los resorts terrestres. Sin embargo, a diferencia del destino -ilimitado, con historia, tradición, vías de comunicación, espacios públicos, viviendas-, el crucero es un espacio limitado y preparado con unas características fijas y herméticas. El crucero ha evolucionado desde el concepto de transporte marítimo hacia una auténtica dimensión turística, en la que se ha llevado a cabo reestructuraciones y adaptaciones a las pautas del mercado.

El puerto, como elemento que permite la movilidad y el intercambio, proporcionando vida a las ciudades (Aguiló, 1999), debe disponer de todos los elementos necesarios para facilitar el embarque y desembarque de pasajeros. Principalmente, terminales, estaciones marítimas, muelles que permitan el acceso y recepción de cruceros de gran tamaño, todos ellos sin barreras, accesibles y adaptadas. En este sentido se hace especial mención a la estación marítima como ítem importante dentro del crucero, no porque pertenezca a él, 
sino porque es el único punto de contacto que tiene con tierra. Además, al igual que el crucero, pero en este caso similar a un aeropuerto, se trata de un no lugar, pues es el espacio en el cual el individuo requiere ser documentado, así como revisado de sus pertenencias, forma parte de un conjunto, de un espacio del que únicamente puede salir siendo identificado (Spielberg, 2004). Así mismo, las terminales de cruceros se entienden por imprescindibles, no sólo por cumplir la función de embarco y desembarco de pasajeros, sino porque son las nuevas puertas de la ciudad y diseñadas para cumplir un función turística. Son pensadas como hitos arquitectónicos que forman parte de ese frente marítimo, modificando en ocasiones el skyline de la ciudad. Estas construcciones se llevan a cabo como infraestructuras modernas con altos estándares de calidad y eficiencia, capaz de contener diversos usos y servicios. Así pues, estamos ante un caso de megaestructura, pues ofrece multiespacio, complejidad y flexibilidad, se produce un intercambio de espacios, sistemas y vías que los interconectan.

Las terminales, al igual que las estaciones de tren y autobús o los aeropuertos, son edificios para el transporte que se incorporan directamente a la calidad de los servicios que la ciudad ofrece tanto a sus habitantes como a los turistas. Son infraestructuras que se estructuran a partir de las circulaciones, de los flujos de entrada y salida de pasajeros, de la conexión con la ciudad y el lugar de embarque o desembarque. Se presentan como un intercambiador entre transporte y ciudad. En el caso de la terminal marítima, entre barco y ciudad, entre mar y tierra, y proporciona así, un nuevo acceso al entorno urbano.

En este contexto se destaca la Terminal Internacional Marítima de Yokohama, realizada por el estudio FOA, una vez ganado el concurso de su proyección en 1994. El edificio se levante como nexo de unión entre el paisaje marítimo y el urbano, y se plantea el descubrimiento de dicha relación según la dirección de circulación del usuario.

\begin{abstract}
"Nuestra propuesta para la nueva terminal será como un dispositivo de mediación entre el sistema de espacios públicos de Yokohama y la gestión del flujo de pasajeros. Un mecanismo para una desterritorialización recíproca: la de un espacio público que rodea la terminal, y la de una estructura funcional que se convierte en el molde de un espacio público atipológico, un paisaje sin instrucciones para ser ocupado" (Zaera-Polo, 2001).
\end{abstract}

Así pues, queda situada como lugar de esparcimiento múltiple con diversas opciones y nuevas visiones.

De esta manera, al igual que el turismo de eventos estimula la renovación urbana, como se ha visto anteriormente, el turismo de cruceros, en muchas ciudades portuarias, ha conllevado y proporcionado efectos positivos que benefician ampliamente, no sólo a los pasajeros de cruceros, sino al público en general: construcción de nuevas viviendas, rehabilitación de edificios, dotación de espacios públicos y zonas verdes, posicionamiento de los lugares de interés cultural, mejora de la imagen de la ciudad y creación de empleo. Dichos efectos, traen consigo, una mejora en la regeneración urbana que aumentará el número de turistas y que implica un desarrollo económico en beneficio de la población local. Por lo que, en este aspecto, también podrían situarse las dos ciudades tratadas por el turismo de eventos, Barcelona, dado que, el mencionado Plan Estratégico, incluía el turismo de cruceros como factor clave en la ciudad, y marcaba su impulso como el prin- 
cipal destino de cruceros en el Mediterráneo (Garay y Cànoves, 2012). Durante la celebración de los Juegos Olímpicos, se utilizaron los cruceros como apoyo a los hoteles de la ciudad. Génova, donde el plan de revitalización del puerto tenía en cuenta: el aumento de su capacidad para hacer frente a los cambios de mercado y el crecimiento del tráfico (directamente relacionado con la creación de empleo), análisis medioambiental y estudio de las infraestructuras e integración en el contexto urbano proporcionando nuevos accesos y revitalización de bienes históricos y culturales (Sánchez, 2003).

El turismo de cruceros, que fomenta el waterfront como espacio sistematizado de intercambio y proporciona elementos sociales, económicos e infraestructurales de una conexión positiva entre el espacio natural del agua y el área urbana construida, puede dar las claves para continuar con los proyectos de éxito de revitalización frente al mar, sin que se produzca la estandarización y la repetición de otros modelos, como ha ocurrido en fases anteriores, que han llevado en algunos casos a la banalización y agotamiento de las fórmulas arquitectónicas (Bruttomesso, 2004).

\subsubsection{El casco histórico. Reactivación para pasajeros de cruceros}

El turismo de cruceros, que fomenta y desarrolla una nueva entrada a la ciudad, donde el flujo de pasajeros (mar-tierra) es opuesto al flujo tradicional del ciudadano (tierra-mar), provoca un cambio de actitud en la relación puerto-ciudad, donde todos los elementos que se involucran en su desarrollo, podrían acercarnos a las escenas y circunstancias que se producían cuando la actividad portuaria compartía el mismo espacio con los ciudadanos que paseaban, llegando a conformar una algarabía callejera muy especial (Alemany, 2009). Por lo que resulta necesario compatibilizar el desarrollo portuario con el desarrollo del recinto urbano, estudiando, analizando y debatiendo todos los componentes existentes: espaciales, urbanísticos, sociales y económicos para que presenten un objetivo común de coexistencia de ambas realidades, aunque por separado, puedan convivir como elementos variables en espacio y tiempo.

En este sentido, las diferentes autoridades tienen que favorecer la sucesión de elementos desde el buque a la ciudad -crucero, puerto, terminal I waterfront, casco histórico- dentro de un sistema integrado de conexión física -flujo de personas continuo-, funcional -mix de usos medioambientalmente integrados- o social -preservación de lo existente- (Andrade et al., 2012). Los autores Andrade, Marín y Blasco (2012), indican esas integraciones para el waterfront, pero podrían extrapolarse a las mejoras de relación mar-tierra mejorando su continuidad.

Así, podría llevarse a cabo la integración física, a través de una conexión accesible, la eliminación de barreras portuarias o la creación de espacios abiertos junto al mar frente a las aglomeraciones del casco histórico. La integración funcional podría encontrarse: en la creación de áreas culturales, lúdicas o comerciales en el frente marítimo al igual que en la ciudad, entrando a formar parte de la trama urbana, incrementando así la participación ciudadana; en la recuperación y mantenimiento del patrimonio histórico generando una red de edificios y espacios interconectados, conservando la idiosincrasia de la ciudad. Este hecho produciría de forma indirecta la integración social, dado que se consolida su historia poniendo en valor su evolución cultural, y la integración social a través de charlas 
formativas y coloquios con la comunidad local mejorarían su comprensión del espacio urbano-portuario y su identificación con el mar, muestra de proyectos para la colaboración del ciudadano que expongan el bienestar de los residentes y exposición de sus experiencias cotidianas para conocer posibles alternativas a la hora de proyectar la ordenación urbanística y arquitectónica del área.

Podría decirse que con el turismo de cruceros, el puerto logra influir en la potenciación de sus zonas adyacentes haciendo atractivo el entorno de forma que se convierte en centro de recepción de nuevos habitantes, afirmando la posibilidad que plantea Sánchez (2003).

Los cruceros ofrecen visitar en un corto periodo de tiempo los destinos de su trayecto, por lo que los pasajeros irrumpen en la ciudad, principalmente en el casco histórico, provocando importantes impactos negativos como el aumento de la capacidad de carga del entorno que amenaza considerablemente el patrimonio cultural y natural, en ocasiones se presentan tensiones asociadas entre la necesidad de un desarrollo económico y la necesidad de mantener el recurso patrimonial (McCarthy, 2004) sin embargo, el éxito de un puerto de cruceros, se debe tanto a su situación geográfica estratégica, como a la disponibilidad de lugares de interés con los que cuente (McCalla, 1998), por lo que, de esta manera, destacan las ciudades portuarias mediterráneas, principalmente, las correspondientes al Sur de Europa, las cuales, presentan una buena situación geográfica, cuenca semicerrada de fácil navegación, que al encontrarse cerca multitud de puertos de cruceros permiten diversificar rutas o proponer nuevas para conocer diferentes enclaves, dado que en la mayoría de los casos, cuenta el propio destino o próximo a él, con Patrimonio de la Humanidad, constituyendo así la mayor garantía internacional de la calidad de su patrimonio. En este aspecto destaca la ciudad griega de Katakolon, que a pesar de su pequeño tamaño, por su estratégica posición geográfica es una parada popular en los cruceros, ofreciendo la visita al enclave arqueológico de Olimpia.

La base de los puertos mediterráneos tradicionales, es el puerto junto a la ciudad, que desde la antigüedad, en casos como Barcelona, Marsella, son puertos base comerciales y ahora también crucerísticos. Este hecho, les permite beneficiarse de la proximidad puertociudad marcando únicamente objetivos de conexión e interrelación.

Por tanto, se deben promover actuaciones de cooperación entre las administraciones, autoridades portuarias, comerciantes y asociaciones para obtener mayor rendimiento económico de las actividades turísticas, mejorar, maximizar y potenciar las conexiones en la interfaz crucero-ciudad permitiendo así el aumento en la calidad de la experiencia del turista, que en el caso del pasajero de cruceros se cree la necesidad de volver a la ciudad.

\section{ESTUDIO DE CASOS. CIUDADES PORTUARIAS MEDITERRÁNEAS}

El importante crecimiento de la actividad crucerística en las ciudades portuarias del mediterráneo, como Barcelona, Venecia, e incluso en los puertos de ciudades como Civitavecchia o El Pireo, puertos de Roma y Atenas respectivamente, está produciendo una nueva oleada de inversiones, tanto en aquellos puertos consolidados que buscan una mejor posición -Marsella, Nápoles, Dubrovnik- como en los puertos que están adentrándose en esta industria y pretenden adquirir un notable reconocimiento -Motril (Puerto de Granada), Valencia-, cuyo objetivo es formar parte de las rutas internacionales de cruceros, dado 
que la zona del Mediterráneo, próspera en conexiones y conectividad debido a la escala de la red de sus ciudades portuarias, presenta un contexto natural, histórico y cultural que le permite reforzar su posición en el mercado de los cruceros (Rodrigue \& Notteboom, 2013). En este sentido, se pueden encontrar tres casos donde la influencia del turismo de cruceros se hace patente en las transformaciones acaecidas en la ciudad.

\subsection{Turismo de cruceros no es factor principal de transformación}

Ciudades como Venecia o Barcelona, que ya son turísticas por sí mismas y capaces de satisfacer la experiencia del crucerista, el crucero no reactiva sus cascos históricos, de hecho, el impacto global de la actividad de cruceros en destinos que son turísticos por naturaleza, se genera un impacto mayor (Chiappa y Abbate, 2013) dado que se unen los propios "turistas terrestres", aumentando los impactos económicos, sociales e infraestructurales positivos o negativos del destino.

Entre los impactos económicos se destaca como positivo su efecto transversal de la actividad en la creación de empleo y el aumento de facturación en sectores como la restauración, el transporte de pasajeros o los operadores turísticos, sin embargo como negativo, se indica que su aportación a la economía de dichas ciudades no es tan importante frente al gasto realizado por otros tipos de turistas o los propios residentes (Seidl et al., 2006; Legoupil, 2013).

En cuanto a los impactos sociales positivos se encuentra la creación de empleo, el intercambio cultural o la valoración del patrimonio local, pudiendo conllevar éste último un impacto social negativo, dado que la masificación de cruceristas puede ocasionar la pérdida del patrimonio, así como el cambio de comercios tradicionales en tiendas de souvenirs o establecimientos de comida rápida, encarecimiento del precio de la vivienda y la emigración de vecinos a zonas más tranquilas de la ciudad evitando la congestión del centro histórico y del tráfico, así como los elevados costes de los productos de primera necesidad que alcanzan precios desorbitados por la localización céntrica del establecimiento dando lugar al proceso de gentrificación.

Por el lado de los impactos infraestructurales, se destaca como positivo las intervenciones ejecutadas con el fin de acercar la ciudad al mar mejorando la relación puerto-ciudad, como negativo se sitúa la pérdida de calidad paisajística por construcciones, pudiendo ocasionar una sobrecarga de los equipamientos, íntimamente relacionado con los impactos ambientales como la contaminación del aire.

En la ciudad de Venecia, se pretendía limitar el acceso de cruceros, dado que se encuentra en un ambiente frágil y los buques cada vez de mayor tamaño, navegan en las proximidades de la plaza San Marcos (Soriani et al., 2009) (figura 2) sin embargo, las autoridades locales han hecho caso omiso y el destino, se encuentra envuelto en una etapa temprana del desarrollo insostenible (Stefanidaki y Lekakou, 2014) existiendo cada vez más rechazo social entre los habitantes, los cuales, bajo la plataforma "No Grandi Navi" pretenden paliar la entrada de cruceros. Esta situación de la ciudad, conocida como "Síndrome de Venecia", puede ocurrir en otras ciudades como Barcelona al existir factores de riesgo como pérdida de residentes en el casco histórico o alta concentración de infraestructuras turísticas (Canalis, 2014). 
Figura 2

NAVEGACIÓN JUNTO A PLAZA SAN MARCOS, VENECIA

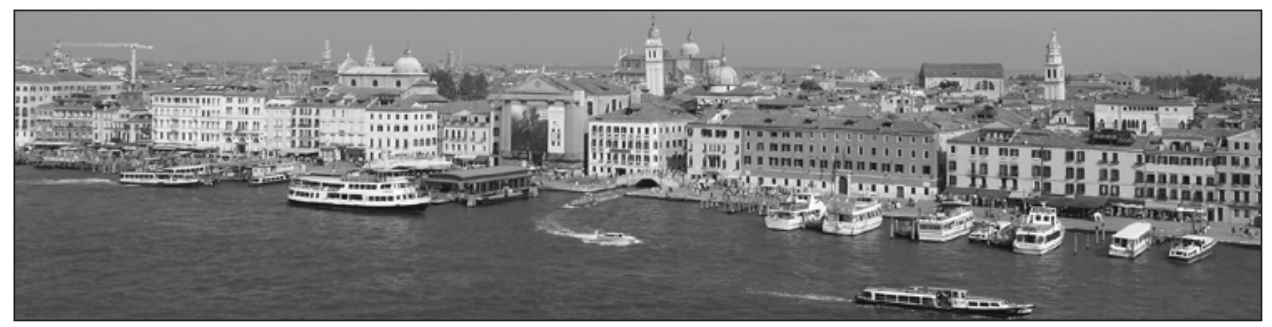

Fuente: Elaboración propia.

En este sentido, las ciudades turísticas que muestran preocupación por la llegada masiva de turistas, siendo los cruceristas el mayor desafío, deben diseñar estrategias responsables que disminuyan la intranquilidad, principalmente social y mantengan la calidad de vida y el bienestar de sus ciudadanos.

\subsection{Turismo de cruceros como un tipo más de turismo que influye en la transfor- mación}

Dubrovnik, presenta un casco histórico que ya es turístico de por sí, por lo que la llegada de cruceros ha beneficiado su inversión en infraestructuras. En 1991, después de la guerra de Yugoslavia, las instalaciones portuarias quedaron dañadas, siendo reconstruido, ampliado y modernizado en el 2009 con el objetivo de atender a los cruceros. Desde entonces se ha convertido en uno de los destinos más importantes y visitados por el turismo de cruceros en el Mediterráneo (Pavlic', 2013) al disponer de recursos culturales y naturales y por disponer de una situación geográfica que le permite ser puerto de escala en las rutas Venecia-Islas Griegas. El puerto, situado junto a una estación de autobuses y demás usos residenciales, se encuentra aproximadamente a 2,5 kilómetros del casco histórico, siendo muy difícil el acceso a pie principalmente porque no se encuentra señalizado. Por tanto, resultaría interesante el desarrollo de una conexión puerto-ciudad del tipo a las intervenciones llevadas a cabo en Marsella para la unión del puerto de cruceros con el casco histórico, pero en vez de realizarse por el frente marítimo, por la propia ciudad.

Las islas griegas también se encontrarían en este caso, atractivas a millones de turistas por sus paisajes naturales y sus playas paradisiacas, ampliaron sus fronteras tras pasar de una economía agrícola a una economía de servicios con el crecimiento de la industria del turismo en la década de los 80' (Fernandes y Pinho, 2015). Los buques, si no hay muelle disponible en puerto, fondean y desplazan a los pasajeros en pequeños barcos hasta tierra. El waterfront es la imagen general de la isla que en muchos casos no existe estación marítima como en los puertos antiguos de Mykonos (figura 3), debajo de la isla, o Santorini, en la bahía de Fira, y el barco fondea situándose lo más próximo al casco histórico. En estos destinos el frente marítimo está íntimamente relacionado con el casco histórico, dado que 
el propio waterfront es parte del casco histórico donde acompaña la arquitectura de los edificios, así como los mismos equipamientos turísticos: cafés, restaurantes, souvenirs y establecimientos de alquiler de coches y motos. Las islas, a pesar de disponer como únicos accesos el aire o el mar y donde el turismo de cruceros supone un beneficio económico local, son más vulnerables sus sistemas naturales que los destinos continentales en cuanto a concentración de personas, actividades e infraestructuras (Fernandes y Pinho, 2015) que podría conllevar a la degradación del paisaje y la construcción descontrolada, perdiendo su idiosincrasia.

\section{Figura 3 \\ WATERFRONT MYKONOS}

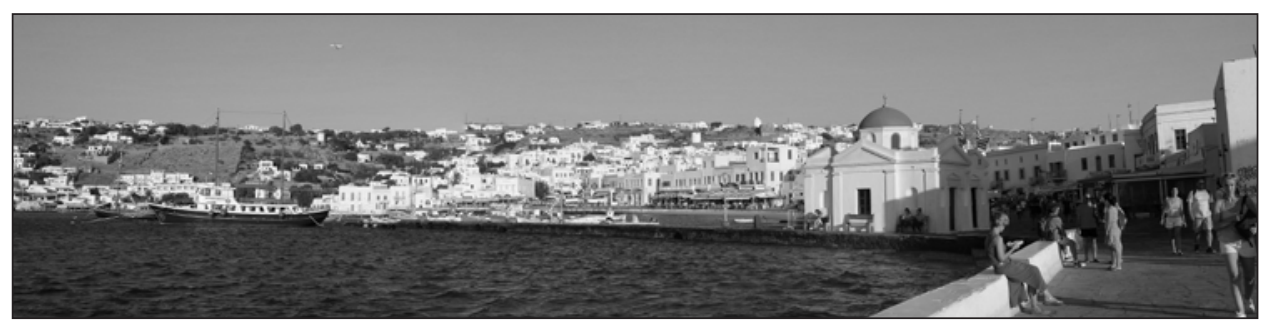

Fuente: Elaboración propia.

Así pues, las ciudades portuarias que se encuentran en este tipo de transformaciones ocasionadas por el turismo de cruceros, son principalmente puertos de escala, como Livorno, que amplía su área cultural ofreciendo otras ciudades como Florencia, Pisa, Lucca o Siena, a menudo puerto base de ciertas compañías, como Túnez o Bari, pero en ningún caso, llegan a ser como los puertos base de Palma de Mallorca o Málaga, dado que sus infraestructuras portuarias, hoteleras, de transporte, tecnológicas, al menos por ahora, no están preparadas para dar servicio a miles de cruceristas.

\subsection{Turismo de cruceros como principal motivo de transformación}

En ciudades como Marsella (Francia), que cambió su estrategia de tráfico de mercancías por la llegada de cruceros turísticos, ha favorecido la mejora de las infraestructuras tanto portuarias como de la ciudad, al rehabilitar la línea de costa existente entre el Viejo Puerto y los muelles de desembarco de los cruceros. Estas actuaciones, junto con otras propuestas incluidas en el proyecto Euroméditerranée (Euromed), contribuyeron a que Marsella fuera elegida Ciudad de la Cultura en 2013.

El proyecto Euromed, lanzado en 1995, abarcaba 480 hectáreas de terreno urbanoportuario. Parte de este proyecto correspondiente a 60 hectáreas, es la Cité de la Méditerranée, la cual empieza en el Viejo Puerto, en el centro de la ciudad y se extiende a lo largo del frente marítimo, unas tres millas, hasta la zona de Bassins-Est donde se sitúan las terminales de crucero. Esta zona ha sido dotada de un gran centro comercial (Terrases du Port), una nueva terminal para cruceros próxima al centro histórico con equipamientos 
públicos, la restauración del edificio Silo d'Arenc como oficinas y sala de conciertos y la creación del MuCEM (Museo de las Civilizaciones de Europa y del Mediterráneo) como proyecto exclusivamente urbano que conlleva 2,6 hectáreas frente a la renovación de la 60 hectáreas en ese espacio (Daamen y Vries, 2013).

Málaga, al igual que Marsella, comenzó a rehabilitar su frente marítimo y con ello, el casco histórico, con la incorporación del turismo de cruceros (figura 4). El puerto genera a su alrededor dos grandes puntos de interés, la ciudad, que aporta momentos culturales y comerciales, y el puerto, con un punto comercial importante, quedando localizada la extensión de influencia generada alrededor del puerto, focalizada en el Muelle Uno y dirigida tanto al pasajero de cruceros como al propio ciudadano. Entre las transformaciones del waterfront, encontramos dos para los cruceros: una, bajo el nombre "El Palmeral de las Sorpresas" contaba con dos edificios culturales y una terminal para los cruceros de menor tamaño y otra, la ampliación del Muelle de Levante, para dar cabida a los mega buques y la construcción de dos terminales. En ese momento, se apostaba por el turismo de cruceros como sector principal para dar vida a ese espacio. Este sector turístico, al ocasionar notables beneficios económicos para la ciudad, ha favorecido la apuesta de Málaga por los pasajeros de cruceros, que directamente influyen en otros tipos de turistas como el cultural y hoy, es una ciudad de museos, ofertando así, diversas actividades al crucerista y el turismo cultural que buscan otros visitantes.

\section{Figura 3 \\ TRANSFORMACIONES EN MÁLAGA PARA EL TURISMO DE CRUCEROS}

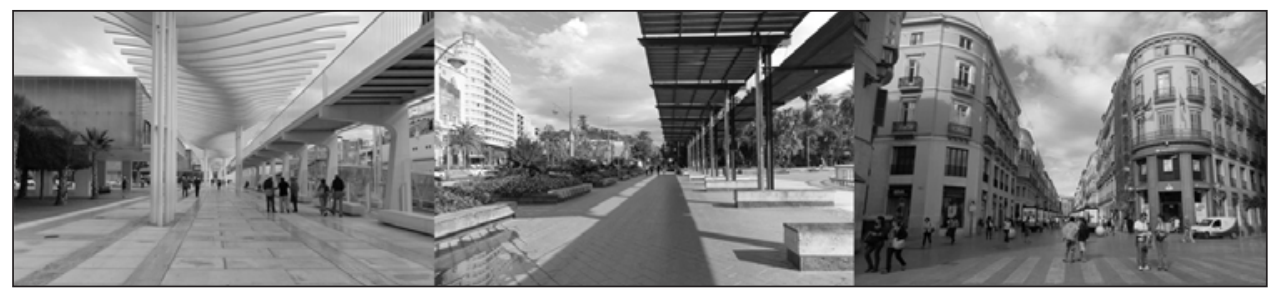

Fuente: Elaboración propia.

Por su parte, la isla de Malta, que sería el caso de Dubrovnik, con la ciudad antigua de La Valetta, Patrimonio de la Humanidad, o como las islas griegas, con la transición a una economía de servicios promovida por el turismo de masas, ha encontrado en el turismo de cruceros un nuevo foco de expansión. Así, con motivo de ser Capital de la Cultura en 2018, su waterfront se encuentra inmerso en un proceso de remodelación, con el objetivo de lograr un cambio positivo en el paseo marítimo a través de la reconstrucción del entorno físico y económico. El desarrollo incluye la restauración de los centros comerciales históricos Forni y Pinto, una terminal de cruceros, plazas y espacios púbicos frente al mar, zonas de almacenamiento para cruceros y actividades turísticas, así como mejoras en la circulación peatonal de vehículos. Acciones que le permitirán competir con los principales destinos de cruceros a nivel internacional. 
La ciudad turca de Kusadasi, al igual que Katakolon, también se ha beneficiado por el mercado crucerista al encontrarse próximo a las ruinas de Éfeso y todas las transformaciones ocasionadas en la ciudad se dirigen a satisfacer las necesidades de los pasajeros de cruceros.

Los puertos dependientes, como Civitavecchia (Roma), El Pireo (Atenas), Motril (Granada), gracias a los cruceros que desembarcan en dichos enclaves para visitar la ciudad principal, han sufrido transformaciones para lograr absorber parte del gasto de los cruceristas. Son básicamente ciudades volcadas en el turismo de cruceros para satisfacer la necesidad del crucerista, acercarlo al principal atractivo turístico.

Podría decirse, que estas ciudades volcadas en el turismo de cruceros en la búsqueda de mayor beneficio económico por parte de otros tipos de turistas, son ciudades que aún no cumplen todas las expectativas de los cruceristas, teniendo que ampliar su área de influencia y acercar a la ciudad otros atractivos turísticos del entorno para promover la calidad y variedad del destino. Así, por ejemplo, desde Marsella se ofrece visitar Avignon o desde Málaga, las cuevas de Nerja. Por lo que, las estrategias de estas ciudades se dirigen a ser por sí solas, destino principal del crucerista sin necesidad de otras alternativas.

\section{EL SMART-DESTINATION COMO ALTERNATIVA}

Tras evaluar las posibilidades de integración en las relaciones crucero-ciudad, dentro de esta nueva etapa del waterfront, se propone una alternativa, dentro de las nuevas pautas de diseño urbanístico y de movilidad, las Smart Cities, los Smart Ports o en este caso, los Smart Cruise Ports (concepto del autor con cierta similitud al proyecto Green Port Cruise propuesto en la región del Mar Báltico (Hamburg Port Authority, 2016)), éstos últimos, desarrollados como parte del factor demanda dentro de los Smart Destinations (Agència Valenciana del Turisme, 2015) dado que a diferencia de la Smart City, el Smart Destination es fomentado principalmente por el sector turístico, en la búsqueda del aumento de su competitividad y la mejora de la experiencia del turista, siendo por tanto, el público objetivo el turista (SEGITTUR, 2015).

Estos conceptos requerirían un estudio más exhaustivo, no obstante, se muestra la idea general del Smart Cruise Pors como punto de partida que podría llevarse a cabo en un Smart Destination, entendiendo éste como un espacio innovador, accesible a todos, apoyado en una estructura tecnológica de vanguardia, que garantice el desarrollo sostenible del territorio turísticos, y facilite la interacción e integración del visitante con el entorno, incrementando la calidad de su experiencia en el destino (SEGITTUR, 2013).

El Smart Cruise Port utilizaría el Smart Destination como eje principal, dado que éste debe contar, entre otros aspectos con: desarrollos tecnológicos -aplicados al turismo (Wifi free, aplicaciones para móviles, códigos QR), a la competitividad de la empresa (gestión de reservas), a la movilidad y el urbanismo (eficiencia de transporte intermodal, gestión del flujo de visitantes en tiempo real), a la sostenibilidad (tecnología led para el alumbrado público), a la seguridad pública, a la sanidad, a la cultura y el patrimonio-, propuestas de innovación que permitan anticiparse al futuro del destino, accesibilidad adecuada a las infraestructuras, equipamientos y a la información (accesibilidad digital) y propuestas de sostenibilidad -medioambientales (reciclaje, uso de energías renovables), 
económicas (puesta en valor del comercio y la industria local), culturales (intercambio de culturas, puesta en valor del patrimonio cultural y arquitectónico)- (SEGITTUR, 2015). Ello daría paso al desarrollo del Smart Cruise Port como plataforma activa utilizada por los pasajeros de cruceros que beneficiaría su acercamiento a la ciudad potenciando una integración física a través de la aplicación de la inteligencia.

El Smart Cruise Port podría desarrollarse en dos partes: una de ellas, una plataforma del puerto de cruceros con las funcionalidades del mismo, los servicios de las terminales, el número de buques atracados, las características de los cruceros, el número de cruceristas, la hora de salida del crucero y que pudiera estar interconectada con la plataforma del Smart Port (en cualquier caso, podría desarrollarse el Smart Cruise Port sin necesidad del Smart Port, que se encuentra vinculado con el tráfico en general, la automatización de controles, los sistemas de seguridad o el control de las emisiones de los barcos (López, 2016), dado que sería exclusivo para el transporte de cruceros) y la otra, una plataforma para la relación crucero-ciudad, desarrollada principalmente para la movilidad inteligente del crucerista, que contara con aplicaciones tecnológicas como recorridos con audiovisuales de elementos patrimoniales, recorridos alternativos al detectar aglomeraciones de personas y congestión en las calles principales garantizando la accesibilidad e informar cuando esté despejada para volver, guía interactiva de restaurantes y cafés geolocalizados con las indicaciones gastronómicas del lugar, que permitan realizar una reserva en tiempo real, conexión directa con los servicios de seguridad y sanitarios.

Además, el desarrollo de un Smart Cruise Port, no sólo se produce a través de una plataforma interconectada, sino que conllevaría un espacio flexible, donde entran en juego empresas, trabajadores, ciudadanos, turistas y cruceristas; social, potenciando la comunicación entre los actores locales implicados y sostenible, manteniendo la calidad del medio en el que se desarrolla la actividad, así como la utilización de energías renovables en la construcción de infraestructuras que beneficiaría el desarrollo social, económico y ambiental.

El destino, debe contar con infraestructuras, planificación que proponga soluciones al impacto puntual y favorezca el futuro de la ciudad apostando por proyectos que fomenten el bien patrimonial, su identidad, accesibilidad, así como la calidad de vida de sus residentes y diversas estrategias de gestión que sean capaces de absorber los impactos. Por lo que, en este sentido el desarrollo de un puerto de cruceros inteligente que aborde una visión estratégica, sostenibilidad, innovación y la adaptación tecnológica de sus recursos, favorecería las necesidades del destino y simplificaría el impacto puntual producido por la masiva llegada de cruceristas al aplicar herramientas como una aplicación móvil que detecta los flujos de personas dirigiendo al turista por rutas alternativas.

\section{CONCLUSIONES}

Tras el estudio realizado, y teniendo en cuenta la evolución del waterfront, cuyos proyectos realizados para la reurbanización de la línea de costa se sitúan íntimamente relacionados con el turismo, siendo uno de los objetivos en la mayoría de las transformaciones (Griffin y Hayllar, 2006) y demostrando ser un importante indicador para que planificadores y políticos dieran forma a "paisajes deseados" (Xie y Gu, 2015), presentados como 
espacios urbanos de gran visibilidad (Airas et al., 2015), se afirma la hipótesis de partida, estamos inmersos en la nueva época del waterfront, donde el turismo de cruceros fomenta la interfaz puerto-ciudad, llegando a convertirse en la interfaz crucero-ciudad, no sólo por llevar incluida y potenciar esa nueva entrada al destino, sino porque principalmente el crucerista, impulsa una reestructuración espacial al formar parte de todos los elementos de la interrelación y es el momento en el que se está prestando especial atención a los elementos puerto y waterfront como parte integrante e insoluble de la ciudad que permite el acercamiento mar-tierra por parte del pasajero y, por su parte, también del residente y turista del opuesto tierra-mar.

En cuanto a las transformaciones ocasionadas en la ciudad portuaria mediterránea por el mercado de cruceros y atendiendo a esta nueva fase del waterfront, se plantean seriamente los problemas de Barcelona o Venecia, poniendo de manifiesto que los diferentes actores se vuelquen, principalmente, en dar solución a los impactos sociales ocasionados. En los casos como Dubrovnik o Livorno donde los cruceros suelen utilizar sus puertos como escala pueden rediseñar sus estrategias para convertirse en puertos base prestando especial atención en la relación crucero-ciudad para generar una conexión sostenible, funcional y atractiva. En las Islas Griegas, la atención se presta en la llamada de atención a los impactos ambientales, sugiriendo la limitación de llegada de cruceros. Por último, las ciudades que se dirigen máxime a los pasajeros de cruceros, deben prestar atención al "Síndrome de Venecia" adecuando sus propuestas a ocasionar los mínimos impactos sociales, ambientales e infraestructurales negativos.

Las actuaciones urbanas y portuarias en las ciudades portuarias con acceso para cruceros deben dirigirse hacia la búsqueda de relación e integración puerto-ciudad, permitiendo que el borde marítimo sea disfrutado por todos. Dichas actuaciones, como obras que puedan modificar sustancialmente la configuración del puerto o alterar de forma notable el medio ambiente, provocando impacto ambiental, podría ser subsanadas mediante medidas correctoras en otros enclaves del puerto o en sus proximidades. En el caso de los cruceristas y su inminente afluencia, pudiendo ocasionar problemas de impacto puntual, las diferentes Autoridades, tienen que prestar especial atención a los accesos puerto-casco histórico, que sean accesibles, los aparcamientos, principalmente de autobuses y taxis, readaptar los horarios comerciales, promover circuitos turísticos con capacidad de absorción y calidad de servicio (Camps, 2009), entre otras cuestiones.

Por tanto, en este último aspecto, la alternativa del Smart Cruise Port en un Smart Destination, propondría recursos que beneficiarían notablemente la relación puerto-ciudad, dado que se desarrollaría como eje canalizador de la interfaz que facilita la movilidad del crucerista, su acercamiento a la ciudad, a los enclaves culturales o territoriales, costumbres o la idiosincrasia del lugar. Su puesta en marcha, sería clave en el desarrollo y caracterización de esta quinta época del waterfront, al disminuir los impactos puntuales ocasionados por el turismo de cruceros

\section{LIMITACIONES Y FUTURAS LÍNEAS DE INVESTIGACIÓN}

Una de las limitaciones encontradas en este artículo es la aplicación de un Smart Cruise Port dentro de un Smart Destination. Se requeriría un análisis más exhaustivo de la Smart 
Destination estudiando los factores que justifican su configuración -tecnología, demanda, eficiencia, competitividad y sostenibilidad-desarrollando una investigación sobre el factor demanda y proponiendo las claves y pautas para formalizar un Smart Cruise Port.

\section{BIBLIOGRAFÍA}

AGÈNCIA VALENCIANA DEL TURISME. INVATUR.TUR (2015): Destinos Turísticos Inteligentes. Manual Operativo para la configuración de Destinos Turísticos Inteligentes. Disponible en: http://www.thinktur.org/media/Manual-de-destinos-tur\%C3\%ADsticosinteligentes.pdf

AGUILÓ, M. (1999): El paisaje construido: una aproximación a la idea de lugar. Madrid, Colegio de Ingenieros de Caminos.

AIRAS, A., HALL, P.V. y STERN, P. (2015): "Asserting historical "distinctiveness" in industrial waterfront transformation», Cities, vol. 44, pp. 86-93.

ALEMANY, J. (1984): El Puerto de Barcelona. Historia y realidad. Barcelona, L'Avenç.

ALEMANY, J. (2009): «Cambios en el transporte marítimo y nueva percepción ciudadana del pasaje portuario», Portus, $\mathrm{n}^{\circ} 18$, pp. 38-43.

ANDRADE MARQUÉS, M. J. (2012): Las transformaciones del puerto de Málaga en el debate de los waterfronts. Mar a la vista. Tesis doctoral. Universidad de Málaga.

ANDRADE, M.J., MARÍN, P. y BLASCO, J. (2012): Puerto-ciudad: estudio comparativo de buenas prácticas: Barcelona, Copenhague, Génova, Gijón, Hamburgo, Helsinki, Málaga, Marsella, Oslo, Sidney, Valencia, Vigo. Málaga, Servicio de Programas Europeos del Ayuntamiento de Málaga.

BARROSO, C., MARTÍN, E. y MARTÍN, D. (2007): «The influence of market hererogeneity on the relationship between a destination's image and tourists' future behaviour», Tourist Management, vol. 28 (1), pp. 175-18.

BROWNILL, S. (1993): Developing London's Docklands. Londres, Paul Chapman.

BROWNILL, S. (2013): «Just Add Water» en The Routledge Companion to Urban Regeneration, 16 de octubre de 2013. London and New York, Routledge. Disponible en: https://www.routledgehandbooks.com/doi/10.4324/9780203108581.ch3

BRUTTOMESSO, R. (2004): «Complejidad en la relación puerto-ciudad», Ingeniería y Territorio, $\mathrm{n}^{\circ}$ 67, pp. 22-31.

CAMPS, S. (2009): «Incidencia del tráfico de cruceros turísticos en las relaciones del puerto y la ciudad de Málaga», Portus, nº 17, pp. 10-15

CANALIS, X. (2014): «Turismo de masas: el riesgo de morir de éxito» en Hosteltur, $\mathrm{n}^{\circ}$ 241, 1 de septiembre de 2014. Disponible en: http://www.hosteltur.com/171500_ turismo-masas-riesgo-morir-exito.html

DEL CHIAPPA, G. y ABBATE, T. (2013): «Island cruise tourism development: a resident's perspective in the context of Italy», Current Issues in Tourism, vol. 19 (13), pp. $1372-1385$.

COOK, I. (2004): «Waterfront Regeneration, Gentrification and the Entrepreneurial State: The Redevelopment of Gunwharf Quays, Portsmouth», en SPA Working Paper 51, junio 2004. Universidad de Manchester. Manchester. Disponible en: http://citeseerx. ist.psu.edu/viewdoc/download?doi=10.1.1.203.4150\&rep=rep1\&type=pdf 
DAAMEN, T.A. y VRIES, I. (2013): «Governing the European port-city interface: institutional impacts on spatial projects between city and port», Journal of Transport Geography, vol. 27, pp. 4-13.

DODMAN, D. (2007): «Post-independence optimism and the legacy of waterfront redevelopment in Kingston, Jamaica», Cities, vol. 24 (4), pp. 273-284.

FERNANDES, R. y PINHO. P. (2017): «The distinctive natura of spatial development on small islands», Progress in Planning, vol. 112, pp. 1-18.

GARAY, L.A., Y CÀNOVES, G. (2012): «Turismo de cruceros en Barcelona. De la marginalidad al liderazgo internacional», Boletín de la Asociación de Geógrafos Españoles, $\mathrm{n}^{\circ} 60$, pp. 253-271.

GASTALDI, F. y CAMERIN, F. (2014): «La regeneración urbana y los grandes eventos en Génova en el período 1992-2004», Revista de Estudios Urbanos y Ciencias Sociales, vol. 5 (1), pp. 71-88.

GOSPODINI, A. (2001): «Urban waterfront redevelopment in Greek cities-a framework for redesigning Space», Cities, vol. 18 (5), pp. 285-295.

GOSPODINI, A. (2006): «Portraying, classifying and understanding the emerging landscapes in the post-industrial city», Cities, vol. 23, pp. 311-330.

GRIFFIN, T. y HAYLLAR, B. (2006): «Historic waterfronts as tourism precincts: An experiential perspective», Tourism and Hospitality Research, vol. 7 (1), pp. 3-16.

GUNAY, Z. y DOKMECI, V. (2012): «Culture-led regeneration of Istanbul waterfront: golden horn cultural valley project», Cities, vol. 29 (4), pp. 213-222.

HAMBURG PORT AUTHORITY (2016): Green Cruise Port: Eröffnungskonferenz in Hamburg für nachhaltige Kreuzfahrt. Hamburg Port Authority, 26 de septiembre de 2016. Disponible en: http://www.hamburg-port-authority.de/de/presse/pressearchiv/ Seiten/Pressemitteilung-26-06-2016.aspx

HAGERMAN, C. (2007): «Shaping neighborhoods and nature: urban political ecologies of urban waterfront transformation in Portland, Oregon», Cities, vol. 24 (4), pp. 285-297.

HARMS, H., (2007): «Changes on the Waterfront-Transfroming Harbour Areas. Comparasion and Evaluation of Waterfront Developments in Two Contexts: San Francisco Bay Area and Hamburg, Germany». Preliminary Presentation at Shrinking Cities Conference, Octubre de 2007. Berkeley, University of California. Disponible en: http://iurd. berkeley.edu/wp/2008-02.pdf

HOYLE, B. (1994): «A rediscovered resource-comparative Canadian perceptions of waterfront redevelopment», Journal of Transport Geography, vol. 2 (1), pp. 19-29.

HOYLE, B. (1998): «Cities and Ports: Development Dynamics on the Port-City Interface» en Land Water Intermodal Terminals. Venecia, Editor Rinio Bruttomesso.

HOYLE, B.S. (1999): «Scale and sustainability: the role of community groups in Canadian port-city waterfront change»., Journal of Transport Geography, no 7 (1), pp. 65-78.

JONES, A. (2007): «On the water's edge: Developing cultural regeneration paradigms for urban waterfronts», Tourism, culture \& regeneration. Wallingford, CABI, pp. 143-150.

KWORTNIK, R.J. (2008): «Shipscape influence on the leisure cruise experience», International Journal of Culture Tourism and Hospitality Research, vol. 2 (4), pp. 289-311.

LAIDLEY, J. (2007): «The ecosystem approach and the global imperative on Toronto's Central Waterfront», Cities, vol. 24 (4), pp. 259-272. 
LEGOUPIL, T. (2013): «Los conflictos que genera el turismo de cruceros en Barcelona y otros puertos mediterráneos», Biblio 3W. Revista Bibliográfica de Geografía y Ciencias Sociales, vol. XVIII, no 1.049 (13), 15 de noviembre de 2013.

LÓPEZ, A. (2016): «Un smartPort capta, registra, tracta i analitza la informació de forma òptima i completa», en Fulls dels Enginyers, 11 de marzo de 2017. Barcelona. Disponible en: http://fullsdelsenginyers.cat/article/smartport-capta-registra-tracta-analitzainformacio-forma-optima-completa

MCCALLA, R.J. (1998): «An investigation into site and situation: Cruise ship ports», Tijdschrift voor Economische en Sociale Geografie, vol. (1), pp. 44-55.

MILLSPAUGH, M. (2003): «The inner harbor story. A model of urban waterfront development, Baltimore's Inner Habor offers and adventure in downtown revitalization», Urban Land, vol. 62 (4), pp. 36-41.

NORCLIFFE, G., BASSETT, K., y HOARE, T. (1996): «The emergence of postmodernim on the urban waterfront-geographical perspectives on changing relationships», Journal of Transport Geography, vol. 4 (2), pp. 123-134.

PAVIA, R. (2011): «Waterfronts. Áreas estratégicas de las ciudades portuarias», Portus, $\mathrm{n}^{\mathrm{o}} 22$, pp. $4-15$

PAVLIC', I. (2013): «Cruise Tourism Demand Forecasting - The Case of Dubrovnik», Tourism \& Hospitality Management, vol.19 (1), pp. 125-142.

ROBERTS, P. (2000): «The evolution, definition and purpose of urban regeneration», en Urban regeneration. Sage. Longon, pp. 9-36.

RODRIGUE, J.-P. y NOTTEBOOM, T. (2013): «The geography of cruises: Itineraries, not destinations», Applied Geography, vol. 38, pp. 31-42.

ROSA-JIMÉNEZ, C. Y PEREA-MEDINA, B. (2016): «Una aproximación al estudio de la influencia del turismo de cruceros en la relación biunívoca puerto-ciudad en el Mediterráneo», PORTUS plus, 6. Disponible en: http://portusonline.org/portusplus/ portusplus-6/economy-and-business-6/

SÁNCHEZ PAVÓN, B. (2003): El futuro de las relaciones puerto ciudad. La Coruña, Instituto Universitario de Estudios Marítimos de la Universidad de La Coruña.

SEGITTUR. SECRETARÍA DE ESTADO DE TELECOMUNICACIONES Y PARA LA SOCIEDAD DE LA INFORMACIÓN A LA SOCIEDAD ESTATAL PARA LA GESTIÓN DE LA INNOVACIÓN Y LAS TECNOLOGÍAS TURÍSTICAS, S.A. (2013): Destinos turísticos inteligentes. Secretaría de Estado de Turismo. España.

Disponible en: http://www.segittur.es/opencms/export/sites/segitur/.content/galerias/descargas/documentos/Presentacin-Destinos-Tursticos-Inteligentes.pdf

SEGITTUR. (2015). Informe destinos turísticos: construyendo el futuro. Secretaría de Estado de Turismo. España. Disponible en http://www.agendadigital.gob.es/planesactuaciones/Bibliotecaciudadesinteligentes/Material\%20complementario/Informe-destinos-turisticos-inteligentes.pdf

SEIDL, A., GUILIANO, F. y PRATT, L. (2006): «Cruise tourism and community economic development in Central America and the Caribbean: The case of Costa Rica», Pasos. Revista de Turismo y Patrimonio Cultural, vol. 4 (2), pp. 213-224.

SINCLAIR, M. T., STABLER, M. J. (1997): The Economics of Tourism. London and New York, Routledge. 
SORIANI, S., BERTAZZON, S., CESARE, F. Y RECH, G. (2009): «Cruising in the Mediterranean: structural aspects and evolutionary trends», Maritime Policy \& Management. The flagship journal of international shipping and port research, vol. 36 (3), pp. 235-251.

SPIELBERG, S. (2004): La terminal (The Terminal) [Película]. Estados Unidos, DreamWorks.

STEFANIDAKI, E. Y LEKAKOU, M. (2014): «Cruise carrying capacity: A conceptual approach», Research in Transportation Business \& Management, vol. 13, pp. 43-52

SUN, X., JIAO, Y. y TIAN, P. (2011): «Marketing research and revenue optimization for the cruise industry: a concise review», International Journal of Hospitality Management, vol. 30 (3), pp. 746-755.

VALLEGA, A. (2001): «Urban waterfront facing integrated coastal management», Ocean \& Coastal Management, vol. 44 (5-6), pp. 379-410.

VAYONA, A. (2011): «Investigating the preferences of individuals in redeveloping waterfronts: the case of the port of Thessaloniki-Greece», Cities, vol. 28 (5), pp. 424-432.

WAKEFIELD, S. (2007): «Great expectations: waterfront redevelopment and the Hamilton Harbour Waterfront Trail», Cities, vol. 24 (4), pp. 298-310.

XIE, P. F. y GU, K. (2015): «The changing urban morphology: Waterfront redevelopment and event tourism in New Zealand», Tourism Management Perspectives, vol. 15, pp. 105-114.

ZAERA-POLO, A. (2001): «Como una montaña rusa» en Verb Processing. Actar. Barcelona. 
